\title{
On contractible curves on normal surfaces
}

\author{
Stefan Schröer
}

February 1, 2008

\begin{abstract}
We give characterizations of contractible curves on proper normal algebraic surfaces in terms of complementary Weil divisors. From this we obtain some generalizations of the classical criteria for contractibility of Castelnuovo and Artin. Furthermore, we will derive a finiteness result on homogeneous spectra defined by Weil divisors on proper normal algebraic surfaces.
\end{abstract}

\section{Introduction}

The goal of this paper is to characterize contractible curves on proper normal algebraic surfaces.

The corresponding question for compact normal complex-analytic surfaces was completely solved by Grauert [8]: A curve $R$ on such a complexanalytic surface $X$ is contractible to another complex-analytic surface $Y$ if and only if the curve $R$ is negative definite. Later, Artin [3] showed that a similar result holds in the category of proper normal 2-dimensional algebraic spaces.

Usually, if one starts with a proper normal algebraic surface $X$ and a negative definite curve $R \subset X$, the resulting algebraic space $Y$ is not a scheme anymore. We want to characterize those curves $R \subset X$ for which $Y$ remains a scheme. For simplicity, we call such curves contractible. Our question was already considered by Artin in a series of two papers [1] [2],

Key words: normal surfaces, contraction, modification.

Mathematics subject classification (1991): 14C20, 14E10, 14J05, 14J17. 
where he gives several necessary or sufficient conditions for smooth surfaces. For example, the classical Castelnuovo criterion tells us that an exceptional curve of the first kind is contractible.

Our main result is a characterization of contractible curves $R \subset X$ in terms of complementary Weil divisors, see theorem (3.4). Roughly speaking, one has to find a Weil divisor which defines the contraction numerically and behaves reasonably on the formal completion $\mathfrak{X}$ of $R \subset X$. From this characterization we will easily derive some generalizations of the classical results of Castelnuovo and Artin. Furthermore, our approach will explain certain differences between characteristic $p>0$ and characteristic zero.

The results will be useful in the classification à la Enriques of proper normal algebraic surfaces, since they allow us to introduce the technique of extremal rays from Mori theory without conditions on the singularities. Since our ground field $K$ is arbitrary, the results might also be applied to generic hyperplane sections or generic fibres in higher dimensional geometry.

As an application we will show that for every Weil divisor $D \in Z^{1}(X)$, the homogeneous spectrum $P(X, D)$ of the graded algebra $\oplus_{n \geq 0} H^{0}(X, n D)$ is always a scheme of finite type. Here the interesting point is that the algebra usually fails to be finitely generated.

This article is divided into six sections, including the introduction. In the second section we will set up the notation and recall some facts about the cone of curves for proper normal surfaces. The third section contains a characterization of almost affine open subsets and our main results on contractible curves. The fourth section is a bit technical. We introduce the notion of numerical $\mathbb{Q}$-factoriality and look for situations in which our criteria are applicable. The fifth section contains some sufficient conditions for contractibility. In the last section we prove that the homogeneous spectra $P(X, D)$ are always schemes of finite type.

I would like to thank the referee for pointing out an error in a preliminary version and several useful suggestions.

\section{The cone of curves}

This section is preparatory in nature. We will set up the notation and discuss the cone of curves for proper normal surfaces. Throughout the paper, we will work over an arbitrary base field $K$. The word surface always refers to a 2- 
dimensional, irreducible, separated $K$-scheme of finite type. In this section, $X$ will always be a proper normal surface.

(2.1) Let $Z^{1}(X)$ be the group of Weil divisors. If $X$ is a proper regular surface, the group of Weil divisors comes along with the $\mathbb{Z}$-valued intersection pairing $A, B \mapsto A \cdot B$. If $X$ is just a proper normal surface, Mumford 21] pointed out that there is still a $\mathbb{Q}$-valued intersection form on $Z^{1}(X)$ with the usual properties. Mumford's approach uses resolutions of singularities for surfaces, which has been established in full generality by Lipman [20].

(2.2) We will call a curve $R \subset X$ negative definite if the intersection matrix $\Phi=\left(R_{i} \cdot R_{j}\right)$ for the irreducible components $R_{i} \subset R$ is negative definite. For example, if $f: X \rightarrow Y$ is a birational morphism of proper normal surfaces, the contracted curve $R \subset X$ is negative definite, see for example [16], X, 1.9. Since $R_{i} \cdot R_{j} \geq 0$ holds for $i \neq j$, it is a well known fact from linear algebra that the inverse matrix $\Phi^{-1}$ has negative entries. Moreover, if $R$ is connected, all entries are strictly negative. We will use this fact several times.

(2.3) A Weil divisor $A \in Z^{1}(X)$ which lies in the radical of the rational intersection pairing is called numerically trivial. Let us write $N(X)$ for the quotient of $Z^{1}(X)$ modulo the radical of Mumford's rational intersection pairing. We obtain a non-degenerate intersection pairing

$$
N(X) \times N(X) \longrightarrow \mathbb{Q} \text {. }
$$

It is well-known that $N(X)$ is a free $\mathbb{Z}$-module of finite type. The Hodge index theorem tells us that this intersection form has exactly one positive eigenvalue. In Mori theory, one traditionally works with the pairing $N^{1}(X) \times$ $N_{1}(X) \rightarrow \mathbb{Z}$ induced by $\operatorname{Div}(X) \times Z^{1}(X) \rightarrow \mathbb{Z}$. But $\operatorname{Pic}(X)=0$ might easily happen if $X$ is non-projective [24], in which case this pairing has lost its significance.

(2.4) We call a subset of a real vector space a cone if it is closed under multiplication with positive scalars and addition. The real vector space $N(X, \mathbb{R})=N(X) \otimes \mathbb{R}$ contains two important cones, which we should recall.

The closed cone $\overline{\mathrm{NE}}(X)$ generated by all curves $C \subset X$ is called the pseudo-effective cone. 
A Weil divisor $A$ meeting the condition of the Nakai criterion for ampleness, which means $A^{2}>0$ and $A \cdot C>0$ for all curves $C \subset X$, will be called ample. The pseudo-ample cone $\overline{\mathrm{NA}}(X)$ is the closed cone generated by the ample cycles. Note that an ample Weil divisor does not define a closed embedding into some $\mathbb{P}^{n}$ unless it is a $\mathbb{Q}$-Cartier divisor.

It follows from [15], XIII, 7.1 that $\overline{\mathrm{NE}}(X)$ is polar to $\overline{\mathrm{NA}}(X)$.

Since the pseudo-effective cone is closed and contains no lines, it is generated by its extremal rays. One calls a closed subcone $P \subset \overline{\mathrm{NE}}(X)$ extremal if $e+e^{\prime} \in P$ for $e, e^{\prime} \in \overline{\mathrm{NE}}(X)$ implies $e, e^{\prime} \in P$. The extremal closed subcones have the following geometric significance:

(2.5) Proposition. The extremal closed subcones $P \subset \overline{\mathrm{NE}}(X)$ are precisely the following subsets:

(i) Cones of the form $P=\sum \mathbb{R}_{+} R_{i}$, generated by the irreducible components $R_{i}$ of a unique reduced negative definite curve $R \subset X$.

(ii) Cones of the form $P=\overline{\mathrm{NE}}(X) \cap F^{\perp}$ for certain real pseudo-effective class $F \in \partial \overline{\mathrm{NA}}(X)$ with $F^{2}=0$.

Moreover, the cones $P=\sum \mathbb{R}_{+} R_{i}$ of the first sort satisfy the following finiteness condition: If $Q \subset N(X, \mathbb{R})$ is the closed cone generated by all irreducible curves $C \subset X$ not supported by $R$, then $P \cap Q=0$ holds.

Proof. An extremal closed subcone $P \subset \overline{\mathrm{NE}}(X)$ is of the form $\overline{\mathrm{NE}}(X) \cap a^{\perp}$ for some support function $a \in \partial \overline{\mathrm{NA}}(X)$. If $a^{2}=0$ holds, we are in case (ii). Assume that $a^{2}>0$ holds. Then the intersection form on the vector subspace $P-P$ is negative definite. Write $P=\sum P_{i}$ as the convex hull of extremal rays. The argument in [19], lemma 4.12 for smooth surfaces also applies for normal surfaces, consequently each $P_{i}$ is generated by a unique integral, negative definite curve $R_{i} \subset X$. If there would be a relation $e_{j}=\sum_{i \neq j} \lambda_{i} e_{i}$, the coefficients must be positive, and we obtain the contradiction

$$
0>R_{j}^{2}=\sum_{i \neq j} \lambda_{i} R_{i} \cdot R_{j} \geq 0 .
$$

So the $R_{i}$ are linearly independent, hence finite in number, and $R=\cup R_{i}$ is the negative definite curve whose irreducible components generate $P$. Conversely, one easily sees that the irreducible components of a negative definite curve generate an extremal cone. 
To verify the finiteness condition, let $e \in P \cap Q$. We have $e=\sum \lambda_{i} R_{i}$ with positive coefficients and $e=\lim C_{n}$ for certain real effective 1-cycles $C_{n}$ whose components are not contained in $R$. Hence we have

$$
0>e^{2}=\lim \left(e \cdot C_{n}\right)=\lim \left(\sum \lambda_{i} R_{i} \cdot C_{n}\right) \geq 0,
$$

contradiction. QED.

(2.6) Remark. It is possible to extend Mumford's intersection pairing fruitfully to unibranched surfaces. Let $X$ be a unibranched surface, which means that the normalization $X^{\prime} \rightarrow X$ is a bijective map [12], EGA 0, 23.2.1. Obviously we have $Z^{1}(X)=Z^{1}\left(X^{\prime}\right)$. If $\eta \in X$ is the generic point, let $d$ be the length of the Artin ring $\mathcal{O}_{X, \eta}$. For two Weil divisors $A, B$ on $X$ the result [13], EGA IV, 21.10.4 forces us to put

$$
A \cdot B=d(A \cdot B)_{X^{\prime}},
$$

where the right hand side is computed on the normalization. The results of this paper remain true for unibranched surfaces, but we are content with the normal case. Nevertheless, it should be noted that from a conceptual point of view, unibranched surfaces form a better category, since this category is stable under extensions of the ground field $K$, which is not true for normal surfaces.

\section{Characterization of contractible curves}

This section contains our main results on contractible curves. Throughout, $X$ will be a proper normal surface. We first make the trivial observation that it suffices to treat the case of connected curves:

(3.1) Lemma. A curve $R \subset X$ is contractible if and only if all its connected components $R_{i} \subset X$ are contractible.

Proof. Use patching. QED.

For the purpose of this paper, the following terminology will be useful. We call an open subset $U \subset X$ almost affine if the affine hull $U^{\text {aff }}=$ Spec $\Gamma\left(U, \mathcal{O}_{X}\right)$ is of finite type over the ground field $K$ and the canonical morphism $U \rightarrow U^{\text {aff }}$ is proper and birational. We remark that these are precisely the semi-affine open subsets of Goodman and Landman [7] with 
a 2-dimensional ring of global sections $\Gamma\left(U, \mathcal{O}_{X}\right)$. There is a kind of dual notion for curves: We say that a curve $A \subset X$ is ample on itself if $A \cdot A_{i}>0$ holds for all irreducible components $A_{i} \subset A$. These concepts are related in the following way:

(3.2) Proposition. Let $U \subset X$ be an open subset, $C=X \backslash U$ its complement, and $C_{i} \subset C$ the integral components. Then the following conditions are equivalent:

a) The open subset $U \subset X$ is almost affine.

b) The closed subset $C \subset X$ is a connected curve and some Weil divisor $A$ supported by $C$ has $A^{2}>0$.

c) There is a curve $A \subset X$ with $\operatorname{Supp}(A)=C$ which is ample on itself.

Proof. We verify the implications $(a) \Rightarrow(b) \Rightarrow(c) \Rightarrow(a)$. Assume that $U$ is almost affine. Passing to the contraction $f: X \rightarrow Y$ defined by $U \rightarrow U^{\text {aff }}$, we can assume that $U$ is affine. Then $\mathcal{O}_{U}$ is ample, and we easily find a section $s \in \Gamma\left(U, \mathcal{O}_{X}\right)$ defining a Cartier divisor $D_{U} \subset U$ whose closure $\overline{D_{U}} \subset X$ intersects each component $C_{i}$. According to 15, II, 2.2.6, each $C_{i}$ is a curve. Multiplying $s$ by a suitable power of $t \in \Gamma\left(U, \mathcal{O}_{X}\right)$ defined by the canonical homomorphism $\mathcal{O}_{X} \rightarrow \mathcal{O}_{X}(C)$ we can assume that the domain of definition $\operatorname{dom}(s) \subset X$ equals $U$. Let $D=\operatorname{div}(s)$ be the corresponding principal divisor on $X$ with $D \cap U=D_{U}$ and write cyc $(D)=D_{1}-D_{2}$ with $D_{1}=\overline{D_{U}}$; then $D_{2} \cdot C_{i}=D_{1} \cdot C_{i}>0$ holds for each component $C_{i}$. Since $\operatorname{dom}(s)=U$, the Weil divisor $D_{2}$ is effective with $\operatorname{Supp}\left(D_{2}\right)=C$. Suppose $C^{\prime} \subset C$ is a connected component. Let $D_{2}^{\prime} \subset D_{2}$ be the corresponding connected component. Then $\left(D_{2}^{\prime}\right)^{2}=D_{2}^{\prime} \cdot D_{2}=D_{2}^{\prime} \cdot D_{1}>0$. Moreover, the curve $C$ is be connected by the Hodge index theorem. Hence $A=D_{2}$ satisfies condition $(b)$.

Now assume that $(b)$ holds. Decompose $A=A_{+}-A_{-}$into positive and negative part. Then $0<A^{2}=A_{+}^{2}-2 A_{+} A_{-}+A_{-}^{2} \leq A_{+}^{2}+A_{-}^{2}$. So there is a curve $A_{0} \subset X$ supported by $C$ with $A_{0}^{2}>0$. Now we rename the irreducible components of $C$ and find a complete list $C_{1}, \ldots, C_{n}$, possibly with repetitions, with $A_{0} \cdot C_{1}>0$ and $C_{i} \cap C_{i+1}$ nonempty. Inductively we define curves $A_{j} \subset X$ with support $A_{0} \cup C_{1} \cup \ldots \cup C_{j}$ and $A_{j} \cdot C_{i}>0$ for $1 \leq i \leq j$ as follows: If $A_{j}$ is already defined, then $A_{j+1}=\lambda_{j} A_{j}+C_{j+1}$ meets our conditions for $\lambda_{j}>0$ sufficiently large. Now $A=A_{n}$ is the desired curve which is ample on itself. 
Finally, assume that $(c)$ holds. Let $g: X^{\prime} \rightarrow X$ be a resolution of all the singularities $x \in \operatorname{Sing}(X) \cap C$. Then $g^{*}(A)$ is an effective $\mathbb{Q}$-Cartier divisor with support $A^{\prime}=g^{-1}(A)$. Let $E^{\prime} \in \operatorname{Div}\left(X^{\prime}\right)$ be a relatively ample divisor whose support is contracted by $g$. Then $A^{\prime}=g^{*}(n A)+E^{\prime}$ is effective with support $A^{\prime}$, provided $n>0$ is sufficiently large, and ample on itself. Hence it suffices to treat the case that $A$ is a Cartier divisor. The associated invertible sheaf $\mathcal{L}=\mathcal{O}_{X}(A)$ is ample on $A$. According to [G], theorem 1.10, a multiple $\mathcal{L}^{\otimes n}$ is globally generated, and the homogeneous spectrum $Y$ of $\Gamma(X, \operatorname{Sym} \mathcal{L})$ yields a birational contraction $f: X \rightarrow Y$. We infer that $U=f^{-1}(V)$ is the preimage of an affine open subset $V \subset Y$, hence is almost affine. QED.

From this we easily derive a geometric characterization of contractible curves in terms of complementary curves ample on themselves:

(3.3) Theorem. A connected negative definite curve $R \subset X$ is contractible if and only if there is a curve $A \subset X$ disjoint to $R$ with $A \cdot C>0$ for every curve $C \subset X$ not supported by $R$.

Proof. Assume there is a contraction $f: X \rightarrow Y$ mapping $R$ to a closed point $y \in Y$. Then the complement $Y \backslash V$ of an affine open neighborhood $V \subset Y$ can be regarded as a curve on $X$. According to [3.2] it is the support of a curve $A \subset X$ ample on itself with the desired property. Conversely, if there is such a curve $A \subset X$, the open subset $U=X \backslash A$ is almost affine and defines the contraction $f: X \rightarrow Y$ of $R$. QED.

We have seen that one can always find a possibly non-effective Weil divisor $A$ satisfying the above condition, and the real problem is to choose an effective one. Our main result has the advantage that it does not involve any conditions of effectivity:

(3.4) Theorem. A connected negative definite curve $R \subset X$ is contractible if and only if there is a Weil divisor $A \in Z^{1}(X)$ satisfying the following three conditions:

(i) The Weil divisor $A$ is Cartier near $R \subset X$.

(ii) We have $A \cdot C \geq 0$ for all curves $C \subset X$, with equality if and only if $C \subset R$ holds.

(iii) For every integer $m>0$ there is an integer $n>0$ and a numerically trivial Weil divisor $N \in Z^{1}(X)$ which is Cartier near $R \subset X$ such that the linear class of $n A+N$ is trivial on $m R$. 
Proof. According to (3.3) the conditions are necessary, and the real issue is to show sufficiency.

We first get rid of the singularities. Let $g: X^{\prime} \rightarrow X$ be a resolution of all singularities $x \in \operatorname{Sing}(X) \cap R$. According to [10], EGA II, 8.11.1 we have to show that $R^{\prime}=g^{-1}(R)$ is contractible. A straightforward argument shows that $A^{\prime}=g^{*}(A)$ satisfies the three conditions on $X^{\prime}$, hence we can assume that $R \subset \operatorname{Reg}(X)$ holds. Now let $g: X^{\prime} \rightarrow X$ be a resolution of all remaining singularities. According to (3.1) we have to show that $R$ viewed as a curve $R^{\prime} \subset X^{\prime}$ is contractible. Let $P^{\prime} \subset N\left(X^{\prime}, \mathbb{R}\right)$ be the cone generated by the irreducible components of $R^{\prime}$ and $Q^{\prime} \subset N\left(X^{\prime}, \mathbb{R}\right)$ the closed cone generated by all other integral curves. Choose a relatively ample exceptional divisor $D^{\prime} \in$ $Z^{1}\left(X^{\prime}\right)$ contracted by $g$. If $U^{\prime} \subset N\left(X^{\prime}, \mathbb{R}\right)$ is a compact neighborhood of zero, the linear form associated to $D^{\prime}$ is bounded on $U^{\prime} \cap Q^{\prime}$. Since $P^{\prime} \cap Q^{\prime}=0$ holds by (2.5), the $\mathbb{Q}$-divisor $A^{\prime}=D^{\prime}+g^{*}(n A)$ is strictly positive on the punctured neighborhood $U^{\prime} \cap Q^{\prime} \backslash\{0\}$ provided $n$ is sufficiently large. Consequently, the numerical class of $A^{\prime}$ is a support function of the pseudo-effective cone with respect to the extremal subcone $P^{\prime}$, and condition $(i)$ holds true on $X^{\prime}$. The other conditions trivially remain unaffected, hence we can assume that $X$ is a regular surface.

Obviously $A^{2}>0$ holds. Since $R$ is negative definite, there exists a divisor $D \in Z^{1}(X)$ supported by $R$ which is anti-ample on $R$; according to $[2.2$, the divisor is effective and its support equals $R$. We have

$$
(t A-D)^{2}=t^{2} A^{2}-2 t A \cdot D+D^{2}>0
$$

for all $t>0$ sufficiently large. Arguing as above we also see that $(t A-D) \cdot C>$ 0 holds for all curves $C \subset Y$, provided $t>0$ is sufficiently large. We now replace $A$ by a suitable multiple and assume, using the Nakai criterion, that $A-D$ is ample.

Set $\mathcal{L}=\mathcal{O}_{X}(A)$ and $\mathcal{I}=\mathcal{O}_{X}(-D)$; thus $\mathcal{L}$ is pseudo-ample and $\mathcal{L} \otimes \mathcal{I}$ is ample. According to Fujita's vanishing result [6], theorem 5.1, there is a natural number $t_{0}>0$ such that

$$
H^{1}\left(X, \mathcal{L}^{\otimes s} \otimes(\mathcal{L} \otimes \mathcal{I})^{\otimes t} \otimes \mathcal{N}\right)=0
$$

holds for all integers $s \geq 0, t \geq t_{0}$ and all numerically trivial invertible sheaves $\mathcal{N}$. If we replace $\mathcal{L}$ and $\mathcal{I}$ by $\mathcal{L}^{\otimes t_{0}}$ and $\mathcal{I}^{\otimes t_{0}}$ we can assume that

$$
H^{1}\left(X, \mathcal{L}^{\otimes s} \otimes \mathcal{I} \otimes \mathcal{N}\right)=0
$$


holds for all integers $s>0$ and all numerically trivial invertible sheaves $\mathcal{N}$. Thus the right hand term in the exact sequence

$$
H^{0}\left(X, \mathcal{L}^{\otimes s} \otimes \mathcal{N}\right) \longrightarrow H^{0}\left(D, \mathcal{L}^{\otimes s} \otimes \mathcal{N} \mid D\right) \longrightarrow H^{1}\left(X, \mathcal{L}^{\otimes s} \otimes \mathcal{I} \otimes \mathcal{N}\right)
$$

is zero. Now choose an integer $m$ with $D \subset m R$. According to condition $(c)$, we can pick a numerically trivial invertible sheaf $\mathcal{N}$ and an integer $n>0$ such that the restriction $\mathcal{L}^{\otimes n} \otimes \mathcal{N} \mid D$ is trivial. Let us replace $\mathcal{L}$ by $\mathcal{L}^{\otimes n} \otimes \mathcal{N}$. Consider the open subset $U \subset X$ on which $\mathcal{L}$ is globally generated; by construction, this open set contains the generic points of $R$, hence $\mathcal{L}$ is ample on the complement $X \backslash U$. According to [6], theorem 1.10, some multiple $\mathcal{L}^{\otimes t}$ is globally generated, and the homogeneous spectrum

$$
Y=\operatorname{Proj}(\Gamma(X, \operatorname{Sym} \mathcal{L}))
$$

is a projective normal surface yielding the desired contraction $f: X \rightarrow Y$ of R. QED.

\section{Improvement of cycles}

How can one ensure that the conditions of theorem [3.4) are fulfilled? It is easy to find a Weil divisor $A \in Z^{1}(X)$ satisfying the condition (ii) of (3.4). In this section we discuss the possibilities to improve the Weil divisor $A$ in such a way that it also satisfies the other two more delicate conditions (i) and (iii). For this purpose the following notion is useful:

(4.1) Definition. Let $S$ be an arbitrary subset of a proper normal surface $X$. We say that $X$ is numerically $\mathbb{Q}$-factorial with respect to $S$ if each Weil divisor $D$ is numerically equivalent to a $\mathbb{Q}$-Weil divisor which is $\mathbb{Q}$-Cartier near $S$.

Of course, only the points $x \in S$ whose local rings $\mathcal{O}_{X, x}$ are not $\mathbb{Q}$-factorial are relevant for this notion. If the conditions holds for $S=X$ we call the surface $X$ numerically $\mathbb{Q}$-factorial. According to the Nakai criterion, such a surface is projective; more precisely, the canonical map $\operatorname{Pic}(X) \rightarrow N(X)$ has finite cokernel.

We have the following behaviour under birational morphisms:

(4.2) Proposition. Let $f: X \rightarrow Y$ be a birational morphism of proper normal surfaces, $R \subset X$ the contracted curve, $\mathfrak{X} \subset X$ the corresponding 
formal completion, $S \subset X$ a subset containing $R$, and $T \subset Y$ its image. If the cokernel of $\operatorname{Pic}^{0}(X) \rightarrow \operatorname{Pic}^{0}(\mathfrak{X})$ is a torsion group and if $X$ is numerically $\mathbb{Q}$-factorial with respect to $S$, then $Y$ is numerically $\mathbb{Q}$-factorial with respect to $T$.

Proof. Given a cycle $D \in Z^{1}(Y)$; then there is an integer $n>0$ and a numerically trivial Weil divisor $N$ on $X$ such that $f^{*}(n D)+N$ is Cartier near $S$. Passing to a multiple and adding a numerically trivial Cartier divisor, we can assume that the corresponding reflexive $\mathcal{O}_{X}$-module $\mathcal{L}$ is trivial on $\mathfrak{X}$. Hence $\mathcal{M}=f_{*}(\mathcal{L})$ is invertible at $T$ and is represented by $n D+f_{*}(N)$. QED.

Let $n R \subset X$ be the infinitesimal neighborhoods of the contracted curve $R$. Then the inverse system of groups $n \mapsto H^{1}\left(R, \mathcal{O}_{n R}\right)$ is eventually constant, and we obtain a well-defined group scheme of finite type $\mathrm{Pic}_{\mathfrak{X} / K}^{0}=\mathrm{Pic}_{n R / K}^{0}$, provided $n$ is sufficiently large. We can use the group scheme $G$ defined by the exact sequence

$$
\operatorname{Pic}_{X / K}^{0} \longrightarrow \operatorname{Pic}_{\mathfrak{X} / K}^{0} \longrightarrow G \longrightarrow 0
$$

to check the hypothesis of the previous result, using the following observation:

(4.3) Lemma. Let $R \subset S$ be a closed subscheme of a proper $K$-scheme S. If $H^{1}\left(S, \mathcal{O}_{S}\right) \rightarrow H^{1}\left(R, \mathcal{O}_{R}\right)$ is surjective, or if the base field $K$ is of characteristic $p>0$ and the cokernel $G$ of the homomorphism $\operatorname{Pic}_{S / K}^{0} \rightarrow$ $\operatorname{Pic}_{R / K}^{0}$ is a unipotent group scheme, then the cokernel of $\operatorname{Pic}^{0}(S) \rightarrow \operatorname{Pic}^{0}(R)$ is a torsion group.

Proof. Let $\mathcal{L}$ be an numerically trivial invertible $\mathcal{O}_{R}$-module and $l \in$ $\operatorname{Pic}_{R / K}^{0}$ the corresponding rational point. The map $H^{1}\left(S, \mathcal{O}_{S}\right) \rightarrow H^{1}\left(R, \mathcal{O}_{R}\right)$ is the tangential map for the homomorphism $\operatorname{Pic}_{S / K}^{0} \rightarrow \operatorname{Pic}_{R / K}^{0}$; if the first condition holds, this map is surjective, and we find a closed point $m \in \operatorname{Pic}_{S / K}^{0}$ mapping to $l$. Assume that the second condition holds. From the definition of unipotent group schemes [14, SGA 3, p. 534, it follows immediately that $G(K)$ is a torsion group. Replacing $\mathcal{L}$ by a multiple we also find a closed point $m \in \operatorname{Pic}_{S / K}^{0}$ mapping to $l$.

If the base field is algebraically closed, the point $m$ is represented by a numerically trivial invertible $\mathcal{O}_{S}$-module $\mathcal{M}$ restricting to $\mathcal{L}$. In general, we find a finite field extension $K \subset K^{\prime}$ and an invertible sheaf $\mathcal{M}^{\prime}$ on $X^{\prime}=$ $X \otimes K^{\prime}$ restricting to $\mathcal{L}^{\prime}=\mathcal{L} \otimes K^{\prime}$ on $R^{\prime}=R \otimes K^{\prime}$. Since $p_{*}\left(\mathcal{O}_{S^{\prime}}\right)$ is a locally 
free $\mathcal{O}_{S}$-module, say of rank $n>0$, there is a commutative diagram of norm homomorphism [10], EGA II, 6.6.8

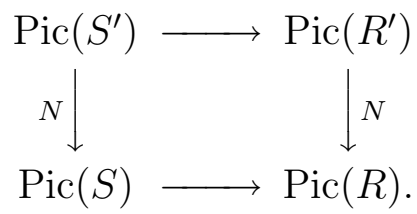

Now $\mathcal{L}^{\otimes n}=N\left(\mathcal{L}^{\prime}\right)$ extends to $\mathcal{M}=N\left(\mathcal{M}^{\prime}\right)$. It remains to check that $\mathcal{M}$ is numerically trivial. Using base change, we can assume that $S$ is an integral curve. Then $\mathcal{M}^{\prime}$ is represented by a Cartier divisor $D^{\prime}$ whose cycle $\sum n_{x^{\prime}} x^{\prime}$ satisfies $\sum n_{x^{\prime}} \operatorname{dim} \kappa\left(x^{\prime}\right)=0$. Obviously, the cycle $\operatorname{cyc}\left(f_{*}(D)\right)$ is of degree zero. QED.

(4.4) Corollary. Let $Y$ be a proper normal surface over a finite ground field $K$ or with $H^{2}\left(Y, \mathcal{O}_{Y}\right)=0$. Then $Y$ is numerically $\mathbb{Q}$-factorial, and in particular projective.

Proof. Let $X \rightarrow Y$ be a resolution of singularities, and $\mathfrak{X} \subset X$ the formal completion of the contracted curve $R \subset X$. If $H^{2}\left(Y, \mathcal{O}_{Y}\right)=0$ holds, then the restriction $H^{1}\left(X, \mathcal{O}_{X}\right) \rightarrow H^{1}\left(\mathfrak{X}, \mathcal{O}_{\mathfrak{X}}\right)$ is surjective, and the claim follows from the above lemma and (4.2). If the ground field $K$ is finite, then $\mathrm{Pic}_{\mathfrak{X} / K}^{0}$ contains only finitely many rational points, and $\operatorname{Pic}^{0}(\mathfrak{X})$ must be a finite group. Consequently, $Y$ is even $\mathbb{Q}$-factorial. QED.

Now we turn to the following situation: Let $R \subset X$ be a curve on a proper normal surface $X$, and $A \in Z^{1}(X)$ a Weil divisor which is already Cartier near $R$ and numerically trivial on $R$. We would like to know whether we can make the linear class of $A$ trivial on $R$. Here the canonical class $K_{X}$ becomes useful:

(4.5) Proposition. Let $A \in Z^{1}(X)$ be Cartier near $R$ and numerically trivial on $R$. If the curve $m R \subset X$ is contained in the base scheme of $K_{X}+m R$, then there is an integer $n>0$ and a numerically trivial Cartier divisor $N$ such that the linear class of $n A+N$ is trivial on the infinitesimal neighborhood $m R$.

Proof. We have an exact sequence

$$
H^{1}\left(X, \mathcal{O}_{X}\right) \longrightarrow H^{1}\left(R, \mathcal{O}_{m R}\right) \longrightarrow H^{2}\left(X, \mathcal{O}_{X}(-m R)\right) \longrightarrow H^{2}\left(X, \mathcal{O}_{X}\right),
$$


and the map on the right is dual to the canonical map $H^{0}\left(X, \mathcal{O}_{X}\left(K_{X}\right)\right) \rightarrow$ $H^{0}\left(X, \mathcal{O}_{X}\left(K_{X}+m R\right)\right)$. Recall that the base scheme of $K_{X}+m R$ is the intersection of all effective curves $C \subset X$ linearly equivalent to this class, hence the map is surjective. We conclude that $H^{1}\left(X, \mathcal{O}_{X}\right) \rightarrow H^{1}\left(R, \mathcal{O}_{m R}\right)$ is surjective, and the claim follows from (4.3). QED.

In positive characteristics the infinitesimal neighborhoods are irrelevant for our problem:

(4.6) Proposition. Let $A \in Z^{1}(X)$ be Cartier near $R$ and trivial on $R$. Assume that the base field $K$ is of characteristic $p>0$. Then the linear class of $p^{m} A$ is trivial on $m R$ for all integers $m>0$.

Proof. We make induction on the integer $m$. Set $\mathcal{I}_{m}=\mathcal{O}_{X}(-m R)$. The exact sequence

$$
0 \longrightarrow \mathcal{I}_{m} / \mathcal{I}_{m+1} \longrightarrow \mathcal{O}_{(m+1) R}^{\times} \longrightarrow \mathcal{O}_{m R}^{\times} \longrightarrow 1
$$

yields an exact sequence

$$
H^{1}\left(R, \mathcal{I}_{m} / \mathcal{I}_{m+1}\right) \longrightarrow \operatorname{Pic}((m+1) R) \longrightarrow \operatorname{Pic}(m R) \longrightarrow 0 .
$$

By induction, $p^{m} A$ is trivial on $m R$. Hence the class of $p^{m} A$ on $(m+1) R$ lies in the image of $H^{1}\left(R, \mathcal{I}_{m} / \mathcal{I}_{m+1}\right)$. Since this group is annihilated by $p$, we conclude that $p^{m+1} A$ is trivial on $(m+1) R$. QED.

\section{Applications}

In this section we will collect some sufficient conditions for contractibility and discuss an example. We assume that $X$ is a proper normal surface, $R \subset X$ is a connected negative definite curve, and denote by $\mathfrak{X} \subset X$ the corresponding formal completion. Our most general criterion is the following:

(5.1) Theorem. Assume that $X$ is numerically $\mathbb{Q}$-factorial with respect to $R$. If the curve $m R \subset X$ is contained in the base scheme of the class $K_{X}+m R$ for all integers $m>0$, or if the base field $K$ is of characteristic $p>0$ and the cokernel of $\mathrm{Pic}_{X / K}^{0} \rightarrow \mathrm{Pic}_{R / K}^{0}$ is a unipotent group scheme, then $R$ is contractible. 
Proof. Let $A$ be a Weil divisor which is a support function of the pseudoeffective cone $\overline{\mathrm{NE}}(X)$ with respect to the extremal subcone $P=\sum \mathbb{R}_{+} R_{i}$ generated by the irreducible components $R_{i} \subset R$. Since $X$ is numerically $\mathbb{Q}$ factorial with respect to $R$, we can assume that $A$ is Cartier near $R$. Given an integer $m>0$. If the first condition holds, we invoke (4.5) and find an integer $n>0$ and a numerically trivial divisor $N \in \operatorname{Div}^{0}(X)$ such that the linear class of $n A+N$ is trivial on $m R$. If the second conditions holds, we first find such $n$ and $N$ such that $n A+N$ is trivial on $R$. Hence $p^{m}(n A+N)$ is trivial on $m R$ by (4.6). Now the claim follows from theorem (3.4). QED.

The following will be useful for the classification of proper normal surfaces of Kodaira dimension $\kappa(X)=-\infty$ :

(5.2) Corollary. If the linear class $K_{X}+m R$ is not effective for all integers $m>0$, or if the base field $K$ is of characteristic $p>0$ and $K_{X}+R$ is not effective, then $R$ is contractible.

Proof. Since $K_{X}+R$ is not effective, the same holds for $K_{X}$, and $H^{2}\left(X, \mathcal{O}_{X}\right)$ must vanish. According to (4.4), the surface $X$ is numerically $\mathbb{Q}$-factorial. If the first condition holds, the base scheme of $K_{X}+m R$ is the whole surface $X$, thus contains $m R$. If the second condition holds, the map $H^{1}\left(X, \mathcal{O}_{X}\right) \rightarrow H^{1}\left(R, \mathcal{O}_{R}\right)$ is surjective, hence $\operatorname{Pic}_{X / K}^{0} \rightarrow \operatorname{Pic}_{R / K}^{0}$ is an epimorphism. The claim now follows from (5.1). QED.

We can generalize the criterion of Castelnuovo-Enriques from regular to normal proper surfaces:

(5.3) Corollary. Assume that $X$ is numerically $\mathbb{Q}$-factorial with respect to $R$. If $R \subset X$ is irreducible with $R \cdot K_{X} \leq 0$, then $R$ is contractible.

Proof. Assume that $m R$ is not in the fixed scheme of $K_{X}+m R$ for some integer $m>0$. Choosing $m$ minimal, we can represent $K_{X}+m R$ by a curve $C \subset X$ not containing $R$. But

$$
0 \geq K_{X} \cdot R=C \cdot R-m R^{2}>0
$$

gives a contradiction. According to (5.1), $R$ is contractible. QED.

(5.4) Remark. For log-terminal surfaces $X$ and $K_{X} \cdot R<0$, this becomes a special case of the contraction theorem from Mori theory ([18], theorem 3.2.1). 
The following is already contained in [1], theorem 2.9:

(5.5) Proposition. Assume that the $K$-surface $X$ is already defined over a subfield $K^{\prime} \subset K$, such that $K^{\prime}$ is a finite field and that $K^{\prime} \subset K$ is the composition of purely transcendental and radical extensions. Then every negative definite curve $R \subset X$ is contractible.

Proof. Let $X^{\prime}$ be the normal surface over $K^{\prime}$ with $X=X^{\prime} \otimes_{K^{\prime}} K$. According to 15], X, 7.17.4, the mapping $Z^{1}\left(X^{\prime}, \mathbb{Q}\right) \rightarrow Z^{1}(X, \mathbb{Q})$ is surjective up to linear equivalence. Hence there is a negative definite curve $R^{\prime} \subset X^{\prime}$ with $R=R^{\prime} \otimes_{K^{\prime}} K$, and we can assume that $K$ is finite. Then $X$ is $\mathbb{Q}$-factorial, and $\operatorname{Pic}^{0}(m R) \subset \operatorname{Pic}_{m R / K}^{0}(K)$ are finite groups. According to (3.4), the curve $R$ is contractible. QED.

(5.6) Example. The case of geometrically ruled surfaces is already instructive. Let $C$ be a normal proper connected curve and $p: X \rightarrow C$ a geometrically ruled surface. Each section $R \subset X$ determines an extension

$$
0 \longrightarrow \mathcal{O}_{C} \longrightarrow \mathcal{E} \longrightarrow \mathcal{L} \longrightarrow 0
$$

with $\mathcal{E}=p_{*}\left(\mathcal{O}_{X}(R)\right)$ and $\mathcal{L}=p_{*}\left(\mathcal{O}_{R}(R)\right)$. Hence we have $X=\mathbb{P}(\mathcal{E})$ and $R=\mathbb{P}(\mathcal{L})$, and $\mathcal{O}_{R}(R) \simeq p^{*}(\mathcal{L}) \mid R$ holds. Assume there is a section with $R^{2}<0$. Let $A$ be a divisor representing $p^{*}(\mathcal{L}) \otimes \mathcal{O}_{X}(-R)$. Then $A$ is trivial on $R$, and in characteristic $p>0$ we deduce from (3.4) and (4.6) that $R$ is contractible. However, the situation is more complicated in characteristic zero. We have

$$
\operatorname{Pic}(2 R)=\operatorname{Pic}(R) \oplus H^{1}\left(C, \mathcal{L}^{\vee}\right) ;
$$

decomposing the class of $A$ into $(0, \alpha)$, one can show that $\alpha$ corresponds to the Yoneda class in $\operatorname{Ext}^{1}\left(\mathcal{L}, \mathcal{O}_{X}\right)$ of the extension $\mathcal{E}$. In characteristic zero, we conclude that $R$ is contractible if and only if the extension $\mathcal{E}$ is split. In this case a splitting $\mathcal{E} \rightarrow \mathcal{O}_{C}$ yields another section $A \subset X$ disjoint to $R$ with $A^{2}>0$, defining the contraction.

\section{A finiteness result for models}

In this section we show that the model of a normal surface $X$ defined by an arbitrary Weil divisor $D$ is a scheme of finite type. This generalizes results of Zariski [25], Proposition 11.5, Fujita [5], p. 235, and Russo [23]. 
(6.1) Let $X$ be a proper normal surface and $D \in Z^{1}(X)$ a Weil divisor. This yields a graded $K$-algebra

$$
R(X, D)=\oplus_{n \geq 0} H^{0}(X, n D)
$$

which in turn defines a homogeneous spectrum

$$
P(X, D)=\operatorname{Proj}(R(X, D)) .
$$

According to [10], EGA II, 3.7.4 the open subset $U \subset X$ of all points $x \in X$ such that there is a homogeneous $s \in R_{+}(X, D)$ with $s(x) \neq 0$ is the largest open subset on which the canonical map $\mathcal{O}_{X} \otimes R(X, D) \rightarrow \oplus_{n \geq 0} \mathcal{O}_{X}(n D)$ defines a morphism $r: U \rightarrow P(X, D)$. We call the scheme $P(X, D)$ the $D$-model of the surface $X$.

Using [9], EGA I, 6.8.2, we see that for each homogeneous element $s \in$ $R_{+}(X, D)$ the open subset $D_{+}(s) \subset P(X, D)$ equals the affine hull $X_{s}^{\text {aff }}=$ $\operatorname{Spec} \Gamma\left(X_{s}, \mathcal{O}_{X}\right)$, hence if $X_{s_{1}} \cup \ldots \cup X_{s_{n}}$ is a covering of $U$, then $D_{+}\left(s_{1}\right) \cup$ $\ldots \cup D_{+}\left(s_{n}\right)$ is a covering of $r(U) \subset P(X, D)$. In this section we will prove the following

(6.2) Theorem. Let $X$ be a proper normal surface, and $D \in Z^{1}(X)$ an arbitrary Weil divisor. Then the model $P(X, D)$ is a separated normal $K$ scheme of finite type of dimension $\leq 2$.

It is well known that the algebra $R(X, D)$ might fail to be finitely generated, see for example [25], p. 562. We will explain the geometric reason for this below. First, we record the following

(6.3) Corollary. Let $U$ be a normal surface, not necessarily proper. Then $\Gamma\left(U, \mathcal{O}_{U}\right)$ is an integrally closed $K$-algebra of finite type and of dimension $\leq 2$.

Proof. By the Nagata compactification theorem [22], we can find a proper scheme $X$ containing $U$ as an open subset. Making a blow-up and a normalization we can assume that $X$ is a proper normal surface and that $D=X \backslash U$ is a Cartier divisor. Now $U^{\text {aff }}$ is an affine open subset of $P(X, D)$, and the claim follows from (6.2). QED.

(6.4) Proof of theorem 6.2. We start with some preliminary reductions. The $D$-model does not change if we replace $D$ by a positive multiple. If 
$H^{0}(X, n D)=0$ holds for all integers $n>0$, then $P(X, D)$ is empty, hence it suffices to treat the case that $D$ is effective. For each $n>0$ let $F_{n} \subset X$ be the fixed curve of $n D$. The effective Weil divisor $M_{n}=n D-F_{n}$ has no fixed curve. Following Kawamata [17], definition 1.1, we call such Weil divisors movable, and refer to $M_{n}$ as the movable part of $n D$. The canonical map $F_{n}: H^{0}\left(X, M_{n}\right) \rightarrow H^{0}(X, n D)$ is bijective, and $M_{n} \cdot C \geq 0$ holds for all curves $C \subset X$. Let $B_{n} \subset X$ be the base scheme of the class $n D$, that is the scheme-theoretical intersection of all curves $C \subset X$ representing the class $n D$. Clearly, $B_{n}$ contains $F_{n}$ and all closed points $x \in X$ where $n D$ is not Cartier. Passing to a multiple of $D$, we can assume that the supports of $B_{n}$ and $F_{n}$ are independent of $n$.

If $M_{n}=0$ hold for all integer $n>0$, we have $P(X, D)=X^{\text {aff }}$, hence we can assume that $D$ has a non-zero movable part.

Now let $F_{n}^{\prime} \subset X$ be the union of all connected components $C \subset F_{n}$ with $C \cdot M_{n}>0$, and let $F_{n}^{\prime \prime}=F_{n}-F_{n}^{\prime}$ be the union of the remaining connected components. Since $M_{n}$ is movable, the condition $M_{n} \cdot F_{n}=0$ is equivalent to $F_{n}^{\prime}=0$. For simplicity, we set $M=M_{1}$ and $F=F_{1}$. The proof of $(6.2)$ will be completed by the next two propositions:

(6.5) Proposition. Under the above assumptions, the scheme $P(X, D)$ is a normal projective curve if and only if $M^{2}=0$ and $M \cdot F=0$ holds.

Proof. Assume that $M^{2}=0$ holds. Then for each curve $\sum \lambda_{i} C_{i} \subset X$ representing $M$ we must have $M \cdot C_{i}=0$. Since $M$ is movable, it can also be represented by a curve $C \subset X$ disjoint to $C_{i}$, and we deduce that $M$ must be a globally generated Cartier divisor. Thus $Y=P(X, M)$ is a proper curve, defining a fibration $f: X \rightarrow Y$, and $M$ is the preimage of an ample class on $Y$.

Now assume that $M \cdot F=0$ also holds; since $M$ is movable, $F^{\prime}$ must vanish. Thus $F=F^{\prime \prime}$ is supported by certain fibres $X_{y}$ of $f: X \rightarrow Y$. Assume that $\operatorname{Supp}\left(F_{y}\right)=\operatorname{Supp}\left(X_{y}\right)$ holds for some point $y \in Y$. Decompose $X_{y}=\sum \lambda_{i} E_{i}$ and $F_{y}=\sum \mu_{i} E_{i}$ into prime cycles. Rearranging the terms, we can assume that $\lambda_{1} / \mu_{1} \geq \lambda_{i} / \mu_{i}$ holds for all indices $i$. We have

$$
\lambda_{1} F_{y}=\mu_{1} X_{y}+\sum_{i>1}\left(\lambda_{1} \mu_{i}-\mu_{1} \lambda_{i}\right) E_{i}
$$

where the first summand $\mu_{1} X_{y}$ is movable, whereas the second summand $\sum_{i>1}\left(\lambda_{1} \mu_{i}-\mu_{1} \lambda_{i}\right) E_{i}$ is effective. Hence $E_{1}$ is not contained in the base 
locus of $\lambda_{1} D$, but by our assumption (6.4), the supports of $F_{n}$ are constant, contradiction. So $\operatorname{Supp}(F)$ contains no fibre of $f: X \rightarrow Y$. Consider the open subset $V=X \backslash F$. I claim that the canonical map $\mathcal{O}_{Y} \rightarrow f_{*}\left(\mathcal{O}_{V}\right)$ is bijective. This is local in $Y$; passing to the henselization $\mathcal{O}_{Y, y} \subset \mathcal{O}_{Y, y}^{\sim}$, we can contract the components $F_{y} \subset X_{y}$, according to [4], Proposition 4, p. 169. By Serre's condition $\left(S_{2}\right)$ we have $f_{*}\left(\mathcal{O}_{V}\right) \otimes \mathcal{O}_{Y, y}^{\sim}=\mathcal{O}_{Y, y}^{\sim}$. Consequently, the open subsets $D_{+}(s)=X_{s}^{\text {aff }}$ form a covering of $Y$ when $s$ ranges over the homogeneous elements of $R_{+}(X, D)$, and we infer $P(X, D)=Y$.

Conversely, assume that $Y=P(X, D)$ is a projective curve, and consider the morphism $r: U \rightarrow Y$, where $U \subset X$ is the maximal open subset as in 6.1). If $M^{2}>0$ holds, then $M \cup F^{\prime}$ supports a curve ample on itself, and its complement is almost affine $[3.2)$; thus $P(X, D)$ would contain a 2dimensional open subset, contradiction. So $M^{2}=0$ holds, and we obtain a fibration $f: X \rightarrow Y$ extending $r$. If $F^{\prime} \neq 0$ holds, the generic fibre $X_{\eta}$ would be affine, contradicting [9], EGA I, 9.3.4. Hence we have $M \cdot F=0$. QED.

(6.6) Proposition. With the assumptions in [6.4], the model $P(X, D)$ is a normal surface if and only if either $M^{2}>0$, or $M^{2}=0$ and $M \cdot F \neq 0$ holds.

Proof. The condition is sufficient: Let $C \subset X$ be a curve representing the class $M+F^{\prime}$ and $V=X \backslash C$. In case $M^{2}>0$ the curve $C$ supports a curve ample on itself. If $M^{2}=0$ holds, $Y=P(X, M)$ is a proper curve, and no connected component of $F^{\prime}$ is vertical with respect to the corresponding fibration $f: X \rightarrow Y$. Again $C$ supports a curve ample on itself. In both cases $V$ is almost affine and $F^{\prime \prime} \subset V$ is contracted in $V^{\text {aff }}$, thus $V^{\text {aff }}$ is an open subset of $P(X, D)$ which is 2-dimensional and of finite type over $K$. According to (6.5), we have $M_{n}^{2}>0$ or $M_{n}^{2}=0$ and $M_{n} \cdot F_{n} \neq 0$ for all $n>0$, consequently $P(X, D)$ is a 2-dimensional scheme of finite type.

Conversely, the condition is necessary by (6.5). QED.

If the scheme $P(X, D)$ is not proper, the algebra $R(X, D)$ is not finitely generated. Concerning this, we have the following characterization:

(6.7) Proposition. Assume that the model $P(X, D)$ is a surface. With the assumptions in (6.4), the surface $P(X, D)$ is proper if and only if $M$ is $\mathbb{Q}$ Cartier and $M \cdot F=0$ holds.

Proof. Assume that $M$ is $\mathbb{Q}$-Cartier and $M \cdot F=0$ holds. Thus $F^{\prime}=0$ and $M^{2}>0$ holds, and $F^{\prime \prime} \subset X$ is a negative definite curve. Let $f: X \rightarrow Y$ be the contraction of the negative definite curve $R \subset X$ orthogonal to $M$. Since 
$M$ is $\mathbb{Q}$-Cartier, $Y=P(X, M)$ holds. Choose homogeneous $s_{1}, \ldots, s_{n} \in$ $R_{+}(X, M)$ with $X=X_{s_{1}} \cup \ldots \cup X_{s_{n}}$ and $R \subset X_{s_{i}}$. Thus we have $U_{s_{i}}^{\text {aff }}=X_{s_{i}}^{\text {aff }}$ and deduce $P(X, D)=P(X, M)$.

Conversely, assume that $Y=P(X, D)$ is a proper surface. Then each curve $C \subset X$ representing the class $M_{n}+F_{n}^{\prime}$ is the support of a curve ample on itself, hence $V=X \backslash C$ is almost affine, and $Y$ is covered by the open subsets $V^{\text {aff }}$. Hence we can extend $r: U \rightarrow Y$ to a proper morphism $r: V \rightarrow Y$ on the larger open subset $V=U \cup F^{\prime \prime}$, thus $X=V$ and $F^{\prime}=0$ hold, and $M \cdot F=0$ follows. Assume that $M$ is not $\mathbb{Q}$-Cartier at some point $x \in X$; then $D$ is also not $\mathbb{Q}$-Cartier at $x$, and we have $x \notin U \cup F^{\prime \prime}$, contradiction. QED.

\section{References}

[1] M. Artin, Some numerical criteria for contractibility of curves on algebraic surfaces. Am. J. Math. 84 (1962), 485-496.

[2] M. Artin: On isolated rational singularities of surfaces. Am. J. Math. 88 (1966), 129-136.

[3] M. Artin: Algebraization of formal moduli II: Existence of modifications. Annals of Math. 91 (1970), 88-135.

[4] S. Bosch, W. Luetkebohmert, M. Raynaud: Néron models. Ergebnisse der Mathematik und ihrer Grenzgebiete (3) 21. Springer, Berlin etc., 1990.

[5] T. Fujita: On L-dimension of coherent sheaves. J. Fac. Sci. Univ. Tokyo 28 (1981), 215-236.

[6] T. Fujita: Semipositive line bundles. J. Fac. Sci. Univ. Tokyo 30 (1983), 353-378.

[7] J. Goodman, A. Landman: Varieties proper over affine schemes. Invent. Math. 20 (1973), 267-312.

[8] H. Grauert: Über Modifikationen und exzeptionelle analytische Mengen. Math. Ann. 146 (1962), 331-368. 
[9] A. Grothendieck, J.A. Dieudonné: Éléments de géométrie algébrique: Le language de schémas. Springer-Verlag, Berlin etc. 1970.

[10] A. Grothendieck, Éléments de géométrie algébrique: Étuede globale élémentaire de quelques classes de morphismes. Publ. Math. IHES 8 (1961).

[11] A. Grothendieck: Éléments de géométrie algébrique: Étuede cohomologique des faiscaux cohérent. Publ. Math. IHES 11 (1961), 17 (1963).

[12] A. Grothendieck: Éléments de géométrie algébrique: Étuede locale des schémas et de morphismes de schémas. Publ. Math. IHES 20 (1967).

[13] A. Grothendieck: Éléments de géométrie algébrique: Étuede locale des schémas et de morphismes de schémas. Publ. Math. IHES 32 (1967).

[14] A. Grothendieck et al.: Schemas en groupes II. Lecture Notes in Math. 152, Springer, Berlin etc., 1970.

[15] A. Grothendieck et al: Théorie des intersections et théorème de Riemann-Roch. Lecture Notes in Math. 225, Springer, Berlin etc., 1971.

[16] A. Grothendieck et al., Groupe de monodromie en géométrie algébrique. Lecture Notes in Math. 340, Springer, Berlin etc., 1973.

[17] Y. Kawamata: On the cone of divisors of Calabi-Yau fiber spaces. Int. J. Math. 8 (1997), 665-687.

[18] Y. Kawamata, K. Matsuda, K. Matsuki: Introduction to the minimal model problem. Algebraic geometry, Sendai 1985, Adv. Stud. Pure Math. 10 (1987), 283-360.

[19] J. Kollár, Rational curves on algebraic varieties. Ergebnisse der Mathematik und ihrer Grenzgebiete. 3. Folge. Band 32. Springer, Berlin etc., 1995.

[20] J. Lipman, Desingularization of two-dimensional schemes. Ann. Math. 107 (1978), 151-207. 
[21] D. Mumford, The topology of normal surface singularities of an algebraic surface and a criterion for simplicity. Publ. Math. IHES 9 (1961), 5-22.

[22] M. Nagata: Imbedding of an abstract variety in a complete variety. J. Math. Kyoto Univ. 2 (1962), 1-10.

[23] F. Russo: On the complement of a nef and big divisor on an algebraic variety. Preprint.

[24] S. Schröer, On non-projective normal surfaces. To appear in Manuscripta Mathematica.

[25] O. Zariski, The theorem of Riemann-Roch for high multiples of an effective divisor on an algebraic surface. Ann. Math. 76 (1962), 560615.

Mathematisches Institut

Ruhr-Universität

44780 Bochum

Germany

E-mail: s.schroeer@ruhr-uni-bochum.de 